DOI: $10.3901 / J M E .2020 .03 .041$

\title{
基于构件-构件邻接矩阵的含复铰 运动链描述与同构判定 ${ }^{*}$
}

\author{
孙伟 孔建益 王兴东 侯 宇 \\ (武汉科技大学机械自动化学院 武汉 430081)
}

\begin{abstract}
摘要: 在运动链型综合过程中, 运动链的同构判定是重要且复杂的问题之一。提出了一种基于构件-构件邻接矩阵的改进 Hamming 数同构判定新方法。针对运动链中复合铰链难以分析和识别问题, 提出了一种构件-构件邻接矩阵的含有复铰运动 链描述方法。该矩阵包含运动链的拓扑结构信息, 具有描述的唯一性。定义改进 Hamming 数原理, 并采用提出的改进 Hamming 数新方法对运动链进行同构判定。案例证明了该新方法的准确性和高效性。
\end{abstract}

关键词：复合铰链；构件-构件邻接矩阵；运动链；同构判定；改进 Hamming 数 中图分类号: TH112

\section{Description and Isomorphism Judgment of the Kinematic Chain with Multiple Joints Based on Link-link Adjacency Matrix}

\author{
SUN Wei KONG Jianyi WANG Xingdong HOU Yu
}

(College of Machinery and Automation, Wuhan University of Technology and Science, Wuhan 430081)

\begin{abstract}
In the synthesis process of kinematic chain, the isomorphism determination of kinematic chain is an important and complicated problem. A new method to improve Hamming number isomorphism determination based on link-link adjacency matrix is proposed. In view of the difficulty in analysis and identification of multiple joint in kinematic chain, a link-link adjacency matrix description method of kinematic chains with multiple joints is proposed. This matrix contains the topological structure information of the kinematic chain and is unique in description. Then, the principle of improve Hamming number is defined, and the proposed new method of improve Hamming number is adopted to identify isomorphism of the kinematic chain with with multiple joints. Finally, the case proves the accuracy and efficiency of the new method.
\end{abstract}

Key words: multiple ltiple joint; link-link adjacency matrix; kinematic chain; isomorphism detection; improve Hamming number

\section{0 前言}

近年来，随着对机构的深入研究，简单的四杆、 五杆机构已经不能满足日以增长的生产需求，多自 由度的机构渐渐进入到我们视野中。由于这类机构 的复杂性和多样性,构型综合研究是非常有必要的。 同构判定又是型综合必不可少步骤之一。在机构拓 扑结构学领域里的最为关键的运动链同构判别问题 在长达 $60 \sim 70$ 年的时间里一直没能得到解决, 这还 使得完全基于计算机的机构自动综合无法实现, 运 动链的拓扑同构判定需要进一步研究。当然, 图的 同构判定广泛应用在各个领域，如模式识别、机器

* 国家自然科学基金资助项目(51875418)。20190323 收到初稿, 20191025 收到修改稿
视觉、分子结构等。同构判定现有方法有很多，例 如最大环路法 ${ }^{[1-3]}$ 、特征值特征矢量法 ${ }^{[4]}$ 、遗传算 法 $^{[5]}$ 、神经网络法 ${ }^{[6-7]}$ 、改进的混合免疫法 ${ }^{[8]}$ 等。但 是这些方法都存在着计算复杂、不够直观、存在适 用范围等缺点。

1964 年，图论理论引入运动链拓扑结构研究领 域，拓扑图以顶点表示构件以边表示关节，建立了 机构简图与拓扑图的关系 ${ }^{[9]}$ 。由于计算机在矩阵计 算的便捷性，图论在机构的研究及发展中提供了一 个有力的数学工具。在运动链型综合中, 无论用哪 种方法, 解决运动链描述的唯一性和全面性问题是 非常重要的, 尤其是含有复铰的运动链。这是一个 在机构拓扑研究领域的瓶颈问题。为了能够对运动 链的复铰描述, 学者们提出了双色拓扑图、矩阵标 识及转换等方法, 特别的是矩阵运算中, 如果将复 
铰看做多元构件, 矩阵的大小都要进行相应的调整。

本文提出了一种构件-构件邻接矩阵描述运动 链新方法, 尤其是对含有复合铰链的运动链的描述, 该方法具有描述运动链结构唯一性。然后, 提出了 改进 Hamming 数同构判定新方法。最后, 通过大量 的案例论证该新方法的准确性和高效性。

\section{1 运动链描述方法}

在运动链分析中运动链的描述扮演着重要的角 色。对于传统的方法, 运动链简图先转换成拓扑图, 然后转换成矩阵。当运动链转换成拓扑图时, 如果 运动链含有复铰, 拓扑图表达是比较麻烦的。

\section{1 试验方法}

平面复合铰链是由多个构件在同一处所组成的 特殊铰链, 简称复铰。在机构分析与综合中, 无论 是运动链拓扑结构描述还是运动链同构识别, 都需 要对复合铰链进行特别的处理。复合铰链是多个构 件在同一处形成的转动副, 通常这些构件之所以能 在同一处组合起来, 是因为构件之间存在一个销轴 类的零件, 将分布于平行平面内的这些构件并联了 起来, 如图 1 所示。
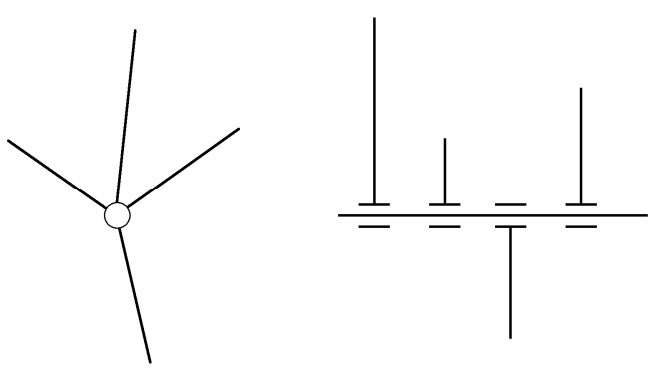

图 1 复合铰链结构示意图

针对机构分析与综合中复合铰链难以处理和含 复铰运动链同构识别困难的问题, 丁华锋等 ${ }^{[10]}$ 采用 一种新的双色拓扑图来表示复铰运动链拓扑结构, 其顶点数目比传统双色图顶点数大为减少。用一种 颜色的实心点 “・” 表示构件, 用另一种颜色的空 心点 “○” 表示运动副, 当构件和运动副关联时, 用边把相应的两个顶点连接起来, 则得到表示平面 复铰运动链的双色拓扑图, 如图 2 所示。YAN 等 ${ }^{[11]}$ 采用缩杆拓扑图来描述和判断带复铰运动链。 $\mathrm{HSU}^{[12]}$ 利用标号树将单个复合铰简化为单铰链串。 刘江南等 ${ }^{[13]}$ 提出了 Pin 构件将复合铰链转化为多 个并联单铰链的新方法, 从而将含复铰运动链转化 为只含单铰的简单运动链。

这些方法具有简单直观的特点, 但不适合计算 机运算。含复铰运动链拓扑描述问题还需要进一步
深入研究, 以寻找更简便、直观、高效的方法。

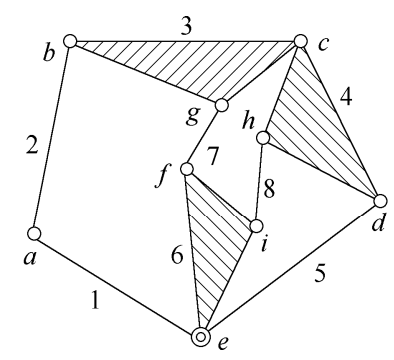

(a) 复铰运动链

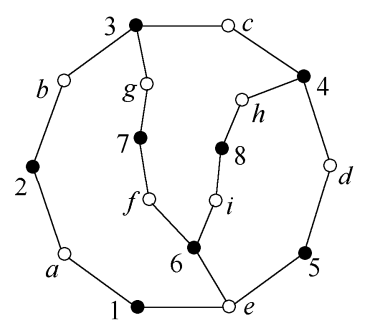

(b) 常规双色拓扑图

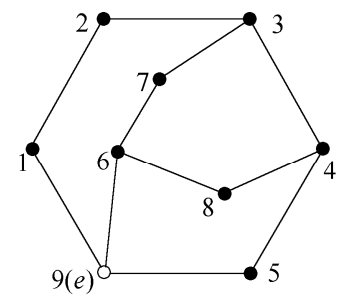

(c) 新双色拓扑图
图 2 复铰运动链及两种双色拓扑图

\section{2 构件-构件链接矩阵}

本文介绍一种构件-构件邻接矩阵实现了含复 铰的运动链直接转成成矩阵。同时构件-构件邻接矩 阵能够唯一表示运动链的拓扑结构。该矩阵的行号 和列号都是构件编号 $L_{n}$ 。矩阵的大小为 $n \times n$ 。 $n$ 表 示运动链的构件数量。运动链的构件-构件邻接矩阵 表示为

$$
\boldsymbol{D}=\left(\begin{array}{ccccccc}
0 & a_{1,2} & \ldots & a_{1, j} & \ldots & a_{1, n-1} & a_{1, n} \\
a_{2,1} & 0 & \ldots & a_{2, j} & \ldots & a_{2, n-1} & a_{2, n} \\
\vdots & \vdots & & \vdots & & \vdots & \vdots \\
a_{i, 1} & \ldots & \ldots & 0 & \ldots & \ldots & a_{i, n} \\
\vdots & \vdots & & \vdots & & \vdots & \vdots \\
a_{n-1,1} & a_{n-1,2} & \ldots & a_{n-1, j} & \ldots & 0 & a_{n-1, n} \\
a_{n, 1} & a_{n, 2} & \ldots & a_{n, j} & \ldots & a_{n, n-1} & 0
\end{array}\right)
$$

式中, 矩阵的对角线的元素 $a_{i, j}(i=j)$ 为 “ 0 ”。矩阵 的其他元素 $a_{i, j}(i \neq j ; i=1, \cdots, n ; j=1, \cdots, n)$ 为关节编 号。如果两个构件之间没有连接, 矩阵的元素的值 为 “ 0 ”。

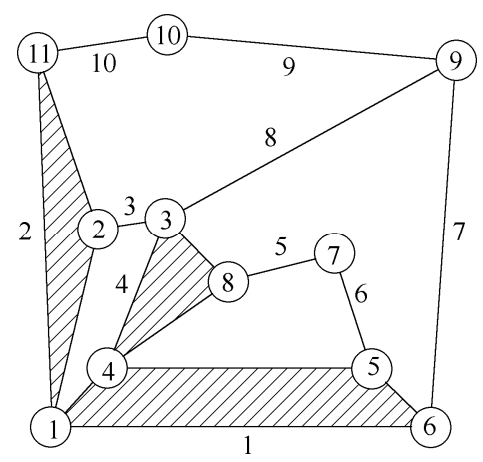

图 3 运动链 $C_{1}$ 的结构简图 
构件编号和关节编号没有任何限制, 只需要按 照顺序标号即可。含 2 个复铰 10 杆运动链 $C_{1}$ 的构 件编号和关节编号如图 3 。运动链 $C_{1}$ 的构件-构件邻 接矩阵表示为

$$
\boldsymbol{D}_{C_{1}}=\left\{\begin{array}{cccccccccc}
0 & 1 & 0 & 4 & 0 & 5 & 6 & 0 & 0 & 0 \\
1 & 0 & 2 & 0 & 0 & 0 & 0 & 0 & 0 & 11 \\
0 & 2 & 0 & 3 & 0 & 0 & 0 & 3 & 0 & 0 \\
4 & 0 & 3 & 0 & 8 & 0 & 0 & 3 & 0 & 0 \\
0 & 0 & 0 & 8 & 0 & 7 & 0 & 0 & 0 & 0 \\
5 & 0 & 0 & 0 & 7 & 0 & 0 & 0 & 0 & 0 \\
6 & 0 & 0 & 0 & 0 & 0 & 0 & 9 & 9 & 0 \\
0 & 0 & 3 & 3 & 0 & 0 & 9 & 0 & 9 & 0 \\
0 & 0 & 0 & 0 & 0 & 0 & 9 & 9 & 0 & 10 \\
0 & 11 & 0 & 0 & 0 & 0 & 0 & 0 & 10 & 0
\end{array}\right\}
$$

构件-构件邻接矩阵 $\boldsymbol{D}_{C_{1}}$ 的元素 $a_{i, j}(i \neq j)$ 表示 运动链 $C_{1}$ 构件 $L_{i}$ 和构件 $L_{j}$ 之间的连接关系 $(i, j=1,2, \cdots, n)$ 。例如, 元素 $a_{4,5}$ 的值是 “ 8 ”, 表示 构件 4 与构件 5 相连接, 连接的关节编号是 8 。同 样地, 构件-构件邻接矩阵 $\boldsymbol{D}_{C_{1}}$ 的第 4 行中, 存在非 零的值 “ 3 ”、“4” 和 “ 8 ”。这表示关节 3 、关节 4 和关节 8 在构件 4 中。其中, 数值 “ 3 ” 出现了 2 次, 表示为该关节是复铰。

\section{2 改进 Hamming 数}

Hamming 数 ${ }^{[14]}$ 定义为任两个 $n$ 维编码位不相 同的位数和, 是在电信领域的一种线性调试码, 以 发明者 RICHARD Hamming 的名字命名。汉明码在 传输的消息流中插入验证码, 当计算机存储或移动 数据时, 可能会产生数据位错误, 以侦测并更正单 一比特错误。由于描述运动链的构件-构件邻接矩阵 元素是关节编号, 不同于传统邻接矩阵元素只有 “ 0 ” 和 “ 1 ”。因此, 本文提出的改进 Hamming(Improved Hamming method, IHM)数方法, 定义如式(3)所示

$$
h_{i j}=\sum_{k=1}^{n} s_{k}, \quad s_{k}=\left\{\begin{array}{lr}
0 & a_{i k}=a_{j k} \\
1 & a_{i k} \neq a_{j k}, a_{i k} a_{j k}=0 \\
i & a_{i k} \neq a_{j k}, a_{i k} a_{j k} \neq 0
\end{array}\right.
$$

式中, $n$ 表示构件-构件矩阵列数, $S_{k}$ 表示第 $k$ 个位 运算结果, 符号 $i$ 表示虚数。例如, 图 3 所示的运 动链 $C_{1}$ 的构件 - 构件邻接矩阵 $\boldsymbol{D}_{C_{1}}$ 中, $h_{13}=0+i+0+i+0+1+1+1+0+0=3+2 i$ 。同样的方 法对矩阵的其他行进行计算可以得到改进的 Hamming 数矩阵 $\boldsymbol{H}=\left[h_{i j}\right]$ 。因此, 10 杆运动链 $C_{1}$ 的改进 Hamming 矩阵为

$$
\boldsymbol{H}_{C_{1}}=\left[\begin{array}{cccccccccc}
0 & 7 & 3+2 i & 8 & 2+2 i & 6 & 7 & 4+2 i & 5+i & 4+i \\
7 & 0 & 6 & 3+2 i & 5 & 3+i & 4+i & 5+i & 4+i & 5 \\
3+2 i & 6 & 0 & 5 & 3+i & 5 & 4+i & 5 & 4+i & 3+i \\
8 & 3+2 i & 5 & 0 & 6 & 2+i & 3+2 i & 6 & 5+i & 6 \\
2+2 i & 5 & 3+i & 6 & 0 & 4 & 5 & 4+i & 5 & 4 \\
6 & 3+i & 5 & 2+i & 4 & 0 & 3+i & 6 & 5 & 4 \\
7 & 4+i & 4+i & 3+2 i & 5 & 3+i & 0 & 5 & 4 & 3+i \\
4+2 i & 5+i & 5 & 6 & 4+i & 6 & 5 & 0 & 5 & 4+i \\
5+i & 4+i & 4+i & 5+i & 5 & 5 & 4 & 5 & 0 & 5 \\
4+i & 5 & 3+i & 6 & 4 & 4 & 3+i & 4+i & 5 & 0
\end{array}\right]
$$

为了更好地用改进 Hamming 数方法表示运动 链特性, 对 Hamming 矩阵 $\boldsymbol{H}_{C_{1}}$ 提出一些相关联的定 义如下。

（1）构件 Hamming 数。构件 $i$ 的构件 Hamming 数为改进 Hamming 矩阵中 $i$ 行所有元素的和。例如, 构件 3 的构件 Hamming 数为 $38+6 i$ 。

(2) 运动链 Hamming 数。运动链的 Hamming 数为所有构件 Hamming 数的和。例如, 运动链 $C_{1}$ 的链 Hamming 数为 $408+52 i$ 。

（3）构件 Hamming 串。构件 $i$ 的构件 Hamming 数为改进 Hamming 矩阵中 $i$ 行元素按照先虚部后实 部从大到小排列, 其中, 将虚数作为上标数。例如, 构件 3 的构件 Hamming 串为 $3^{2}, 4^{1}, 4^{1}, 3^{1}, 3^{1}, 6,5,5,5,0$ 。

根据上述规则, 运动链 $C_{1}$ 的构件 Hamming 数、 运动链 Hamming 数和构件 Hamming 串为

$\operatorname{Total}\left(C_{1}\right): 408+54 i$

1: $46+8 i, 4^{2}, 3^{2}, 2^{2}, 5^{1}, 4^{1}, 8,7,7,6,0$

4: $44+7 i, 3^{2}, 3^{2}, 2^{2}, 5^{1}, 8,6,6,6,5,0$

$8: 44+5 i, 4^{2}, 5^{1}, 4^{1}, 4^{1}, 6,6,5,5,5,0$

2: $42+6 i, 3^{2}, 5^{1}, 4^{1}, 4^{1}, 3^{1}, 7,6,5,5,0$

9: $42+4 i, 5^{1}, 5^{1}, 4^{1}, 4^{1}, 5,5,5,5,4,0$

$7: 38+6 i, 3^{2}, 4^{1}, 4^{1}, 3^{1}, 3^{1}, 7,5,5,4,0$

$3: 38+6 i, 3^{2}, 4^{1}, 4^{1}, 3^{1}, 3^{1}, 6,5,5,5,0$

$5: 38+4 i, 2^{2}, 4^{1}, 3^{1}, 6,5,5,5,4,4,0$

$6: 38+4 i, 2^{2}, 3^{1}, 3^{1}, 6,6,5,5,4,4,0$

$10: 38+4 i, 4^{1}, 4^{1}, 3^{1}, 3^{1}, 6,5,5,4,4,0$ 
式(5)中的构件 Hamming 数排列进行了从大到小排列。

\section{3 同构判定}

改进 Hamming 数同构判定方法具体流程：首 先, 获得运动链的构件-构件邻接矩阵。如果矩阵的 大小是不一样的, 那么运动链就不同构。然后, 通 过改进 Hamming 算法获得运动链的构件 Hamming 数、运动链链 Hamming 数和构件 Hamming 串。如 果信息是相同的, 那么运动链同构。如果任何一个 信息不同，运动链就不同构。

含复铰 10 杆运动链 $C_{2}$ 和 $C_{3}$ 如图 4 所示。在第 1 节中运动链对应关的构件-构件邻接矩阵描述见式 (6)、(7)

$$
\boldsymbol{D}_{C_{2}}=\left\{\begin{array}{cccccccccc}
0 & 1 & 0 & 5 & 6 & 0 & 0 & 7 & 0 & 0 \\
1 & 0 & 3 & 0 & 0 & 2 & 2 & 0 & 0 & 0 \\
0 & 3 & 0 & 4 & 0 & 0 & 0 & 0 & 0 & 0 \\
5 & 0 & 4 & 0 & 0 & 0 & 0 & 0 & 0 & 0 \\
6 & 0 & 0 & 0 & 0 & 11 & 0 & 0 & 0 & 11 \\
0 & 2 & 0 & 0 & 11 & 0 & 2 & 0 & 0 & 11 \\
0 & 2 & 0 & 0 & 0 & 2 & 0 & 8 & 0 & 0 \\
7 & 0 & 0 & 0 & 0 & 0 & 8 & 0 & 9 & 0 \\
0 & 0 & 0 & 0 & 0 & 0 & 0 & 9 & 0 & 10 \\
0 & 0 & 0 & 0 & 11 & 11 & 0 & 0 & 10 & 0
\end{array}\right\}
$$

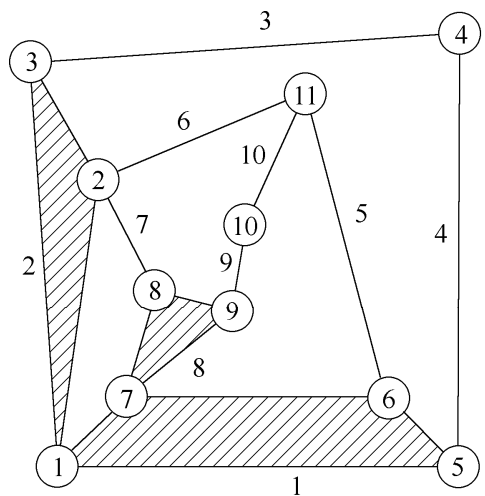

(a) 运动链 $C_{2}$ 结构简图

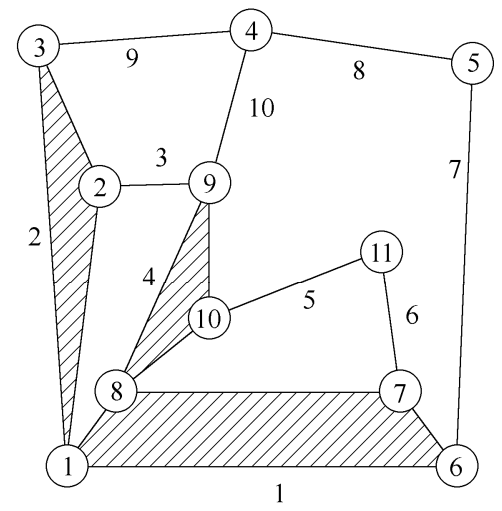

(b) 运动链 $C_{3}$ 结构简图

图 4 运动链 $C_{2}$ 和 $C_{3}$

$$
\boldsymbol{D}_{C_{3}}=\left\{\begin{array}{cccccccccc}
0 & 1 & 0 & 8 & 0 & 7 & 6 & 0 & 0 & 0 \\
1 & 0 & 2 & 0 & 0 & 0 & 0 & 0 & 3 & 0 \\
0 & 2 & 0 & 9 & 0 & 0 & 0 & 0 & 0 & 9 \\
8 & 0 & 9 & 0 & 10 & 0 & 0 & 0 & 0 & 9 \\
0 & 0 & 0 & 10 & 0 & 11 & 0 & 0 & 0 & 0 \\
7 & 0 & 0 & 0 & 11 & 0 & 0 & 0 & 0 & 0 \\
6 & 0 & 0 & 0 & 0 & 0 & 0 & 5 & 0 & 0 \\
0 & 0 & 0 & 0 & 0 & 0 & 5 & 0 & 4 & 4 \\
0 & 3 & 0 & 0 & 0 & 0 & 0 & 4 & 0 & 4 \\
0 & 0 & 9 & 9 & 0 & 0 & 0 & 4 & 4 & 0
\end{array}\right\}
$$

运动链 $C_{2}$ 和 $C_{3}$ 的构件 Hamming 数、链 Hamming 数和构件 Hamming 串为

$$
\operatorname{Total}\left(C_{2}\right): 408+54 i
$$

1: $46+8 i, 4^{2}, 3^{2}, 2^{2}, 5^{1}, 4^{1}, 8,7,7,6,0$

$2: 44+7 i, 3^{2}, 3^{2}, 2^{2}, 5^{1}, 8,6,6,6,5,0$

$6: 44+5 i, 4^{2}, 5^{1}, 4^{1}, 4^{1}, 6,6,5,5,5,0$

$8: 42+6 i, 3^{2}, 5^{1}, 4^{1}, 4^{1}, 3^{1}, 7,6,5,5,0$

$10: 42+4 i, 5^{1}, 5^{1}, 4^{1}, 4^{1}, 5,5,5,5,4,0$

$5: 38+6 i, 3^{2}, 4^{1}, 4^{1}, 3^{1}, 3^{1}, 7,5,5,4,0$

$7: 38+6 i, 3^{2}, 4^{1}, 4^{1}, 3^{1}, 3^{1}, 6,5,5,5,0$

$3: 38+4 i, 2^{2}, 4^{1}, 3^{1}, 6,5,5,5,4,4,0$

$4: 38+4 i, 2^{2}, 3^{1}, 3^{1}, 6,6,5,5,4,4,0$

9: $38+4 i, 4^{1}, 4^{1}, 3^{1}, 3^{1}, 6,5,5,4,4,0$

$\operatorname{Total}\left(C_{3}\right): 408+54 i$

1: $48+7 i, 3^{2}, 2^{2}, 6^{1}, 5^{1}, 5^{1}, 8,7,6,6,0$

$4: 44+7 i, 3^{2}, 2^{2}, 5^{1}, 5^{1}, 4^{1}, 8,6,6,5,0$

$10: 44+5 i, 3^{2}, 6^{1}, 4^{1}, 4^{1}, 6,6,5,5,5,0$

$8: 42+4 i, 5^{1}, 5^{1}, 4^{1}, 4^{1}, 5,5,5,5,4,0$

$2: 40+7 i, 3^{2}, 3^{2}, 4^{1}, 3^{1}, 3^{1}, 7,6,6,5,0$

$9: 40+5 i, 2^{2}, 5^{1}, 5^{1}, 3^{1}, 6,5,5,5,4,0$

$3: 38+6 i, 3^{2}, 2^{2}, 4^{1}, 3^{1}, 6,5,5,5,5,0$

$5: 38+4 i, 2^{2}, 4^{1}, 3^{1}, 6,5,5,5,4,4,0$

$6: 38+4 i, 2^{2}, 3^{1}, 2^{1}, 6,6,5,5,5,4,0$

$7: 36+5 i, 4^{1}, 4^{1}, 3^{1}, 3^{1}, 2^{1}, 6,5,5,4,0$

从式(5)、(8)、(9)中可以看出, 运动链 $C_{1} 、 C_{2}$ 和 $C_{3}$ 的运动链 Hamming 数是一样的, 都是 $408+54 i$ 。 但是运动链 $C_{3}$ 的构件 Hamming 数和构件 Hamming 串与运动链 $C_{1} 、 C_{2}$ 是不一样的。所以可以判断出运 动链 $C_{1}$ 和 $C_{2}$ 是同构运动链, 运动链 $C_{3}$ 与其是非同 构运动链。 


\section{4 案例分析}

采用改进 Hamming 数同构判定方法判断两个 12 杆运动链是否同构, 如图 5 所示。

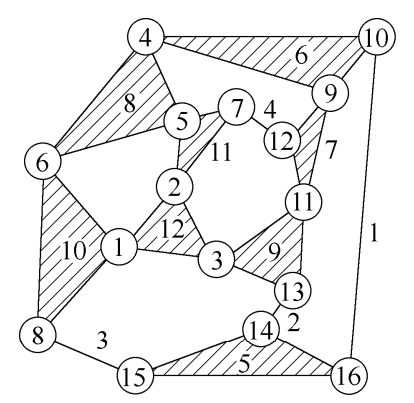

(a) 运动链 $A$

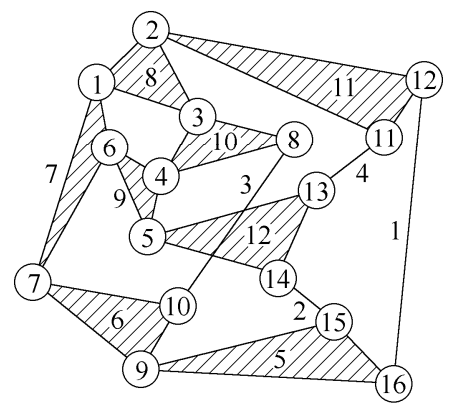

(b) 运动链 $B$

图 512 杆运动链机构简图

运动链 $A$ 和 $B$ 的构件-构件邻接矩阵为

$$
\begin{aligned}
\boldsymbol{D}_{A} & =\left\{\begin{array}{cccccccccccc}
0 & 0 & 0 & 0 & 16 & 10 & 0 & 0 & 0 & 0 & 0 & 0 \\
0 & 0 & 0 & 0 & 14 & 0 & 0 & 0 & 13 & 0 & 0 & 0 \\
0 & 0 & 0 & 0 & 15 & 0 & 0 & 0 & 0 & 8 & 0 & 0 \\
0 & 0 & 0 & 0 & 0 & 0 & 12 & 0 & 0 & 0 & 7 & 0 \\
16 & 14 & 15 & 0 & 0 & 0 & 0 & 0 & 0 & 0 & 0 & 0 \\
10 & 0 & 0 & 0 & 0 & 0 & 9 & 4 & 0 & 0 & 0 & 0 \\
0 & 0 & 0 & 12 & 0 & 9 & 0 & 0 & 11 & 0 & 0 & 0 \\
0 & 0 & 0 & 0 & 0 & 4 & 0 & 0 & 0 & 6 & 5 & 0 \\
0 & 13 & 0 & 0 & 0 & 0 & 11 & 0 & 0 & 0 & 0 & 3 \\
0 & 0 & 8 & 0 & 0 & 0 & 0 & 6 & 0 & 0 & 0 & 1 \\
0 & 0 & 0 & 7 & 0 & 0 & 0 & 5 & 0 & 0 & 0 & 2 \\
0 & 0 & 0 & 0 & 0 & 0 & 0 & 0 & 3 & 1 & 2 & 0
\end{array}\right\} \\
\boldsymbol{D}_{B} & =\left\{\begin{array}{cccccccccccc}
0 & 0 & 0 & 0 & 16 & 0 & 0 & 0 & 0 & 0 & 12 & 0 \\
0 & 0 & 0 & 0 & 15 & 0 & 0 & 0 & 0 & 0 & 0 & 14 \\
0 & 0 & 0 & 0 & 0 & 10 & 0 & 0 & 0 & 8 & 0 & 0 \\
0 & 0 & 0 & 0 & 0 & 0 & 0 & 0 & 0 & 0 & 11 & 13 \\
16 & 15 & 0 & 0 & 0 & 9 & 0 & 0 & 0 & 0 & 0 & 0 \\
0 & 0 & 10 & 0 & 9 & 0 & 7 & 0 & 0 & 0 & 0 & 0 \\
0 & 0 & 0 & 0 & 0 & 7 & 0 & 1 & 6 & 0 & 0 & 0 \\
0 & 0 & 0 & 0 & 0 & 0 & 1 & 0 & 0 & 3 & 2 & 0 \\
0 & 0 & 0 & 0 & 0 & 0 & 6 & 0 & 0 & 4 & 0 & 5 \\
0 & 0 & 8 & 0 & 0 & 0 & 0 & 3 & 4 & 0 & 0 & 0 \\
12 & 0 & 0 & 11 & 0 & 0 & 0 & 2 & 0 & 0 & 0 & 0 \\
0 & 14 & 0 & 13 & 0 & 0 & 0 & 0 & 5 & 0 & 0 & 0
\end{array}\right\}
\end{aligned}
$$

运动链 $A$ 和 $B$ 的构件 Hamming 数、运动链 Hamming 数和构件 Hamming 串为

$\operatorname{Total}(A): 592+56 i$

$$
\begin{gathered}
5: 56+3 i, 4^{1}, 4^{1}, 4^{1}, 6,6,6,6,5,5,5,5,0 \\
10: 52+5 i, 2^{2}, 4^{1}, 4^{1}, 4^{1}, 6,6,6,5,5,5,5,0 \\
11: 52+5 i, 2^{2}, 4^{1}, 4^{1}, 4^{1}, 6,6,6,5,5,5,5,0 \\
6: 52+5 i, 4^{1}, 4^{1}, 4^{1}, 4^{1}, 3^{1}, 6,6,6,5,5,5,0 \\
7: 52+5 i, 4^{1}, 4^{1}, 4^{1}, 3^{1}, 3^{1}, 6,6,6,6,5,5,0 \\
9: 52+5 i, 4^{1}, 4^{1}, 4^{1}, 4^{1}, 3^{1}, 6,6,6,5,5,5,0 \\
8: 50+6 i, 2^{2}, 4^{1}, 3^{1}, 3^{1}, 3^{1}, 6,6,6,6,6,5,0 \\
12: 50+6 i, 2^{2}, 4^{1}, 3^{1}, 3^{1}, 3^{1}, 6,6,6,6,6,5,0 \\
4: 44+4 i, 3^{1}, 3^{1}, 3^{1}, 3^{1}, 5,5,5,5,4,4,4,0 \\
1: 44+4 i, 3^{1}, 3^{1}, 2^{1}, 2^{1}, 5,5,5,5,5,5,4,0 \\
2: 44+4 i, 3^{1}, 3^{1}, 2^{1}, 2^{1}, 5,5,5,5,5,5,4,0 \\
3: 44+4 i, 3^{1}, 3^{1}, 2^{1}, 2^{1}, 5,5,5,5,5,5,4,0
\end{gathered}
$$

$\operatorname{Total}(B): 592+56 i$

$11: 54+4 i, 4^{1}, 4^{1}, 4^{1}, 4^{1}, 6,6,6,5,5,5,5,0$

$12: 54+4 i, 4^{1}, 4^{1}, 4^{1}, 4^{1}, 6,6,6,5,5,5,5,0$

5: $54+4 i, 4^{1}, 4^{1}, 4^{1}, 3^{1}, 6,6,6,6,5,5,5,0$

$10: 52+5 i, 2^{2}, 4^{1}, 4^{1}, 4^{1}, 6,6,6,5,5,5,5,0$

$6: 52+5 i, 4^{1}, 4^{1}, 4^{1}, 3^{1}, 3^{1}, 6,6,6,6,5,5,0$

$7: 50+6 i, 2^{2}, 4^{1}, 4^{1}, 4^{1}, 3^{1}, 6,6,6,5,5,5,0$

8: $50+6 i, 2^{2}, 4^{1}, 3^{1}, 3^{1}, 3^{1}, 6,6,6,6,6,5,0$

9: $50+6 i, 2^{2}, 4^{1}, 3^{1}, 3^{1}, 3^{1}, 6,6,6,6,6,5,0$

3: $44+4 i, 3^{1}, 3^{1}, 3^{1}, 3^{1}, 5,5,5,5,4,4,4,0$

1: $44+4 i, 3^{1}, 3^{1}, 2^{1}, 2^{1}, 5,5,5,5,5,5,4,0$

$2: 44+4 i, 3^{1}, 3^{1}, 2^{1}, 2^{1}, 5,5,5,5,5,5,4,0$

$4: 44+4 i, 3^{1}, 3^{1}, 2^{1}, 2^{1}, 5,5,5,5,5,5,4,0$

从式(12)、(13)来看运动链 $A$ 和 $B$ 的运动链 Hamming 数是一样的。但是运动链 $A$ 的构件 Hamming 数跟运动链 $B$ 是不一样的。例如, 运动链 $A$ 构件 5 的构件 Hamming 数为 $56+3 i$, 运动链 $B$ 的构件 Hamming 数中不存在。因此, 两个 12 杆运 动链是不同构的运动链。

\section{5 结论}

(1) 本文提出了一种新颖的运动链描述方法, 构件-构件邻接矩阵相比于传统邻接矩阵, 能够表示 含复铰运动链的拓扑机构唯一性。实现了运动链的 
简图与构件-构件邻接矩阵一一对应关系。

（2）改进的 Hamming 数同构判定相对于其他同 构判定方法, 该方法不需要复杂的计算, 该方法具 有计算简单和高效性。

\section{参 考 文 献}

[1] DING Huafeng, HUANG Zhen. The establishment of the canonical perimeter topological graph of kinematic chains and isomorphism identification[J]. Journal of Mechanical Design, 2007, 129(9): 915-923.

[2] DING Huafeng, HUANG Zhen. A new theory for the topological structure analysis of kinematic chains and its applications[J]. Mechanism \& Machine Theory, 2007, 42(10): 1264-1279.

[3] DING Huafeng, HUANG Zhen. Isomorphism identification of graphs: Especially for the graphs of kinematic chains[J]. Mechanism and Machine Theory, 2009, 44(1): 122-139.

[4] CHANG Zongyu, ZHANG Ce, YANG Yuhu, et al. A new method to mechanism kinematic chain isomorphism identification[J]. Mechanism \& Machine Theory, 2002, 37(4): 411-417.

[5] YANG Ping, ZENG Kehan. A high-performance approach on mechanism isomorphism identification based on an adaptive hybrid genetic algorithm for digital intelligent manufacturing $[\mathrm{J}]$. Engineering with Computers, 2009, 25(4): 397-403.

[6] GALAN-MARIN G , MERIDA-CASERMEIRO E , LOPEZ-RODEIFUEZ D. Improving neural networks for mechanism kinematic chain isomorphism identification[M]. Kluwer Academic Publishers, 2007.

[7] GALAN-MARIN G , LOPEZ-RODEIFUEZ D , MERIDA-CASERMEIRO E. A new multivalued neural network for isomorphism identification of kinematic chains $[\mathrm{J}]$. Journal of Computing and Information Science in Engineering, 2010, 10(1): 1-4.

[8] YANG Ping, ZENG Kehan, LI Chunquan, et al. An improved hybrid immune algorithm for mechanism kinematic chain isomorphism identification in intelligent design[J]. Soft Computing, 2015， 19(1): 217-223.

[9] MRURHYUNJAYA T S. Kinematic structure of mechanisms revisited[J]. Mechanism \& Machine Theory, 2003, 38(4): 279-320.

[10] 丁华锋, 黄真. 平面机构统一拓扑描述模型的建立及同 构判别 [J]. 机械工程学报，2009，45(3): 99-103.

DING Huafeng, HUANG Zhen. Uniform topological representation model of planar mechanisms and isomorphism identification[J]. Journal of Mechanical Engineering, 2009, 45(3): 99-103.

[11] YAN H S, HSU C H. Contracted graphs of kinematic chains with multiple joints[J]. Mathematical \& Computer Modelling, 1988, 10(9): 681-695.

[12] HSU C H. Enumeration of basic kinetic chains with simple and multiple joints[J]. Journal of the Franklin Institute, 1992, 329(4): 775-789.

[13] 刘江南, 于德介. 基于转化邻接矩阵的含复铰运动链描 述与同构识别 $[\mathrm{J}]$. 机械工程学报, 2012, 48(5): 15-21. LIU Jiangnan, YU Dejie. Representations \& isomorphism identification of planar kinematic chains with multiple joints based on the converted adjacent matrix[J]. Journal of Mechanical Engineering, 2012, 48(5): 15-21.

[14] RAO A C, RAJU D V. Application of the hamming number technique to detect isomorphism among kinematic chains and inversions[J]. Mechanism \& Machine Theory, 1991, 26(1): 55-75.

作者简介: 孙伟, 男, 1990 年出生, 博士, 讲师。主要研究方向为机构 学与机器人。

E-mail:sw35812@gmail.com

王兴东(通信作者), 男, 1970 年出生, 博士, 教授。主要研究方向为机 构学、机器视觉。

E-mail: wangxingdong@wust.edu.cn 